\title{
Practical Experience with Anticoagulation Therapy in an Elderly Polymorbid Rehabilitation Population: What did we Learn?
}

Helen Schmidt ${ }^{12^{*}}$, Felix Angst ${ }^{1}$, Andreas R Gantenbein ${ }^{1,3}$, Susanne Lehmann ${ }^{1}$, André Aeschlimann ${ }^{1}$ and Jürg-Hans Beer ${ }^{3,4}$

${ }^{1}$ RehaClinic, Baden and Bad Zurzach, Switzerland

${ }^{2}$ Psychiatric University Hospital, Zurich, Switzerland

${ }^{3}$ University of Zurich, Zurich, Switzerland

${ }^{4}$ Department of Medicine, Cantonal Hospital of Baden, Switzerland

"Corresponding author: Helen Schmidt, RehaClinic Bad Zurzach, Research Unit, Quellenstrasse 34, CH-5330 Bad Zurzach, Switzerland, Phone: +41562695397; Fax: +41562695170; E-mail: helen.schmidt@uzh.ch

Rec date: Mar 26, 2016; Acc date: May 16, 2016; Pub date: May 18, 2016

Copyright: (02016 Schmidt H, et al. This is an open-access article distributed under the terms of the Creative Commons Attribution License, which permits unrestricted use, distribution, and reproduction in any medium, provided the original author and source are credited.

\begin{abstract}
Objective: The aim of this cross-sectional study was to analyze the prescription patterns of oral anticoagulation and antiplatelet therapy in an elderly population in rehabilitation hospitals.

Methods: 200 patients, after undergoing knee/hip surgery or after a stroke, were evaluated over a time span of one year.

Results: All patients necessitated anticoagulation; the average number of medications taken was 8 . The most frequent combination was the new oral anticoagulation with antiplatelet agents. $35 \%$ of the patients on this combination therapy did not receive proton pump inhibitors.

Conclusion: The increased use of the new oral anticoagulation medicine increases the frequency of dual therapy. Careful and repetitive investigation of indications, benefits and negative side-effects should be considered in order to reduce complications.
\end{abstract}

Keywords: New oral anticoagulation; NOACs; Dual anticoagulation; Elderly population; Rehabilitation

\section{Abbreviations \\ APA: Antiplatelet Agents; APT: Antiplatelet Therapy; LMWH: Low Molecular Weight Heparin; NOAC: New Oral Anticoagulation; NSAID: Non-Steroidal Anti-Inflammatory Drugs; OAC: Oral Anticoagulation; PPI: Proton Pump Inhibitors; SSRI: Selective Serotonin Re-Uptake Inhibitor; VKA: Vitamin K Antagonist}

\section{Background}

Anticoagulant prophylaxis and therapy has become an established cornerstone in clinical medicine and prevents thromboembolism resulting from reduced mobility due to various diseases (American College of Chest Physicians Guidelines 2012) [1]. Antiplatelet agents (APA) are established for the secondary prevention of recurrent strokes or heart attacks. New oral anticoagulants (NOAC) have been introduced, and a solid knowledge regarding their use and their limitations is presupposed [2].

The prevention of venous thromboembolism after total hip or knee arthroplasty by using NOACs was improved as compared to LMWH [3].

The duration of hospitalization has also been decreased, and fewer surgical complications were observed with rivaroxaban [4]. However, consolidated knowledge about their use and limitations has gaps in prescribing providers [1].

The dual anticoagulation therapy is the combination of two agents that influence the blood clotting. It can be the combination of a new oral anticoagulation with a low molecular weight heparin or an antiplatelet agent as well as warfarin instead of NOAC.

Dual anticoagulation increases the need for regular, critical evaluation of benefits and risks, especially the risk of bleeding [5]. Using NOACs in combination with APAs substantially increases the risk of bleeding [6]. The optimal length of such a combined treatment with respect to risk and benefit is not yet known [7]. High-risk combinations may call for gastric protection [8].

Dual anticoagulation therapy is the strongest predictor for nuisance bleeding [5]. Nuisance bleeding is divided by the Bleeding Academic Research Consortium into five types, whereby in Type 1 there is no need to seek medical attention [9].

Every patient on dual therapy should be considered as a recipient of prophylactic PPI to confront the increased risk of gastrointestinal bleeding $[10,11]$.

Because the proportion of elderly, female, polymorbid patients is increasing due to the demographic development, these persons bear a higher risk for complications, caused, for example, by bleeding, or by drug interactions due to polymedication. Polymorbid patients have two or more chronic diseases and complex treatments are needed [12]. 
Several studies have shown that antiplatelet, anticoagulants, diuretics, and NSAIDs (non-steroidal anti-inflammatory drugs) are responsible for more than half of the preventable drug-related admissions to hospital $[13,14]$. Elderly patients also have a high risk of drug-related problems, such as delirium or renal failure, with an increasing number of medications [15].

Drug-drug interactions often occur during hospitalization while patients are under permanent supervision rather than in outpatient settings [16]. As a consequence, critical screening of medication is crucial in elderly, polymorbid, patients receiving multiple drug treatment [15].

Rivaroxaban was introduced in Switzerland in March 2012, as the first NOAC, with the following indications: prophylaxis of deep vein thrombosis (DVT), which may lead to pulmonary embolism (PE) in patients undergoing knee or hip replacement surgery; reduction of the risk of stroke and systemic embolism in patients with non-valvular atrial fibrillation; and the treatment of DVT and PE.

Due to its being the first and due to its possible advantages in the treatment of patients (no need for blood tests, orally available, several indications) its use has increased $[17,18]$.

The aim of the study was to analyze the prescription pattern of OAC and APA, particularly focusing on indication, diagnosis, comorbidities, co-medications and assessment of bleeding risk, in a setting of inpatient rehabilitation with an emphasis on the elderly patients after acute hospitalization.

\section{Methods}

\section{Study population and data collection}

We conducted a cross-sectional study with 200 patients, who were consecutively referred to, at the Rehabilitation Centre RehaClinic in Bad Zurzach and Baden over a time span of one year, in collaboration with the acute teaching hospital in Baden, which treats 19,000 inpatients and more than 100,000 outpatients per year.

The study was approved by the local ethics committee (Health Department Aarau, Switzerland, EK AG 2008/026). Patients were included if they were over 75 years of age, had a stroke or knee/hip replacement, and were treated with OAC or APA therapy.

The population was selected during the first year after the introduction of rivaroxaban to the Swiss market (from March 2012 to March 2013).

Discharge reports (from rehabilitation) were reviewed for diagnoses, medications, comorbidities (cardiovascular history, thromboembolic events and general risk factors for bleeding), and serum creatinine ( $\mathrm{ml} / \mathrm{min}$, calculated by the Cockcroft formula).

Resident doctors from the rehabilitation hospitals wrote these reports according to a structured clinic intern scheme.

They were then reviewed by the authors of this article. Anticoagulation therapy was defined as oral anticoagulation with Vitamin K antagonists (VKA), NOAC or low molecular weight heparin (LMWH). NOACs were used in a lower prophylactic dose (rivaroxaban $10 \mathrm{mg} / \mathrm{d}$ ) after knee or hip replacement, or in a higher, therapeutic dose (rivaroxaban $20 \mathrm{mg} / \mathrm{d}$ ) for the prevention of stroke.

Antiplatelet therapy consisted of therapy with aspirin $(100 \mathrm{mg} / \mathrm{d})$, clopidogrel $75 \mathrm{mg} / \mathrm{d}$, prasugrel, ticagrelor or combination with aspirin.

\section{Analysis}

Descriptive statistic methods were used to characterize patients, diagnoses, and medication. To analyze the bleeding risk, we applied the HAS-BLED score: $\mathrm{H}=$ Hypertension, $\mathrm{A}=\mathrm{Abnormal}$ renal or liver function (1 point each), S=post Stroke, B=Bleeding, L=Labile coagulation parameter (INR), E=Elderly ( $>65$ years), $\mathrm{D}=$ use of Drugs (anticoagulant agents) or alcohol (1 point each). A HAS-BLED score of $\geq 3$ points reflects a risk of bleeding of $5.8 \%$ or higher, reflecting a major bleeding risk [19].

For the indication of anticoagulation in atrial fibrillation, we calculated the CHA2DS2-VASc score: $\mathrm{C}=$ Congestive heart failure, $\mathrm{H}=$ Hypertension, $\mathrm{A}=$ Age $(>75=2$ points $), \mathrm{D}=$ Diabetes mellitus, $\mathrm{S}=$ Stroke/TIA (2 points), V=Vascular disease, A=Age: 65-74 years, $\mathrm{Sc}=$ Sex category (women $>65$ years $=1$ point) [20]. Microsoft Excel and statistic program " $\mathrm{R}$ " were used for all analyses.

\section{Results}

Data sets of 200 patients were analyzed (Table 1). The median age at hospitalization was $81 \pm 4.5$ years (range $75-98$ ), and 66\% were women $(\mathrm{n}=132)$. Forty-five $(25 \%)$ had a stroke, and 151 (75\%) had hip or knee arthroplasty (Table 2).

The mean duration of rehabilitation was $23.7 \pm 10.0$ days. Besides the main diagnosis, $44 \%$ of the patients had a cardiovascular history, and $55 \%$ had an increased bleeding risk by calculation of the HASBLED Score ( $>3$ points, Table 3 ).

The most frequent combination was NOAC prophylactic use with APA (49 patients), followed by APA with LMWH (22 patients).

In the discharge letter from rehabilitation, the indication for treatment was reported in 17/18 (94\%) for therapeutic NOAC, 98/98 (100\%) for prophylactic NOAC, $34 / 39(87 \%)$ for VKA, and $36 / 94$ (38\%) for APA.

On average, $8.1 \pm 2.7$ medications were prescribed on entry, and 8.2 \pm 2.7 at discharge from rehabilitation, with almost no differences between stroke and arthroplasty (Table 1).

Monotherapy (OAC or APA) was administered in 119/200 (59\%) of the subjects, of those 50 (42\%) received NOAC.

Dual therapy was prescribed in $81 / 200$ (41\%) patients. There was no case where a patient received a triple therapy of anticoagulants. The most frequent combination was prophylactic NOAC with APA in 49/81 (60\%), while 4/81 (5\%) had VKA plus APA.

While 55/81 (65\%) received PPI-prophylaxis, a total of 41 patients had a HAS-BLED score of $\geq 3$ points. Twenty-six out of these 41 (63\%) received no PPI, while 10 had a dual oral anticoagulation.

Atrial fibrillation was diagnosed in 46/200 (23\%) cases, 12 patients were treated with therapeutic NOAC, 24 with VKA, and 10 patients did not receive anticoagulation at all.

Twenty-five cases were on prophylactic treatment with rivaroxaban $10 \mathrm{mg} / \mathrm{d}$, with a creatinine clearance between $49 \mathrm{ml} / \mathrm{min}$ and $15 \mathrm{ml} /$ min. One patient had a clearance of less than $30 \mathrm{ml} / \mathrm{min}$, receiving rivaroxaban at a dose of $20 \mathrm{mg} / \mathrm{d}$, which was too high. 
Citation: Schmidt H, Angst F, Gantenbein AR, Lehmann S, Aeschlimann A, et al. (2016) Practical Experience with Anticoagulation Therapy in an Elderly Polymorbid Rehabilitation Population: What did we Learn?. J Gerontol Geriatr Res 5: 301. doi:10.4172/2167-7182.1000301

Page 3 of 5

\begin{tabular}{|c|c|c|c|c|}
\hline & Total & Stroke & Knee Arthroplasty & Hip Replacement \\
\hline $\mathrm{n}$ & 200 & 49 & 54 & 97 \\
\hline Women (n, \%) & $132(66 \%)$ & $22(45 \%)$ & $37(68.5 \%)$ & $73(75.25 \%)$ \\
\hline Age (years) & $81.5 \pm 4.5$ & $81.2 \pm 4.6$ & $80.8 \pm 4.7$ & $82.3 \pm 4.2$ \\
\hline Length of stay (days) & $23.7 \pm 10.0$ & $31.9 \pm 13.3$ & $21.4 \pm 5.9$ & $20.9 \pm 7.3$ \\
\hline Number of drugs at entry & $8.1 \pm 2.7$ & $8.3 \pm 2.8$ & $8.0 \pm 2.1$ & $8.2 \pm 3.0$ \\
\hline Number of drugs at discharge & $8.2 \pm 2.7$ & $8.6 \pm 2.5$ & $8.2 \pm 2.9$ & $7.9 \pm 2.7$ \\
\hline \multicolumn{5}{|l|}{ Comedication } \\
\hline NSAIDs & 19 & 0 & 13 & 6 \\
\hline Corticosteroids & 16 & 1 & 4 & 9 \\
\hline SSRI & 22 & 13 & 4 & 5 \\
\hline \multicolumn{5}{|l|}{ Anticoagulation } \\
\hline NOAC & 115 & 10 & 44 & 61 \\
\hline APA & 41 & 22 & 5 & 14 \\
\hline Vitamin $\mathrm{K}$ & 31 & 15 & 4 & 12 \\
\hline \multirow[t]{2}{*}{ LMWH } & 13 & 2 & 1 & 10 \\
\hline & 19 & 0 & 13 & 6 \\
\hline
\end{tabular}

Table 1: Patients' characteristics and comedication.

\begin{tabular}{|c|c|c|c|c|c|c|c|c|}
\hline Patients with stroke $(\mathrm{N}=49)$ & NOAC prophylactic & NOAC therapeutic & APA & LMWH & VKA & Mono & Dual & $\begin{array}{l}\text { Mono \& } \\
\text { Dual }\end{array}$ \\
\hline NOAC prophylactic & 0 & 0 & 0 & 0 & 0 & & 0 & 0 \\
\hline NOAC therapy & & 10 & 0 & 0 & 0 & & 0 & 10 \\
\hline APA & & & 15 & 5 & 2 & & 7 & 22 \\
\hline LMWH & & & & 1 & 1 & & 1 & 2 \\
\hline VKA & & & & & 15 & & & 15 \\
\hline Total & & & & & & 41 & 8 & 49 \\
\hline $\begin{array}{l}\text { Hip and Knee Arthroplasty } \\
(N=151)\end{array}$ & NOAC prophylactic & NOAC therapeutic & APA & LMWH & VKA & Mono & Dual & $\begin{array}{l}\text { Mono \& } \\
\text { Dual }\end{array}$ \\
\hline NOAC prophylactic & 50 & 0 & 49 & 0 & 0 & & 49 & 99 \\
\hline NOAC therapy & & 5 & 1 & 0 & 0 & & 1 & 6 \\
\hline APA & & & 0 & 17 & 2 & & 19 & 19 \\
\hline LMWH & & & & 7 & 4 & & 4 & 11 \\
\hline VKA & & & & & 16 & & & 16 \\
\hline Total & & & & & & 78 & 73 & 151 \\
\hline
\end{tabular}

Table 2: Drug indications and combinations in the two groups: patients with stroke and patients after Hip/Knee Arthroplasty. 
Complications during rehabilitation occurred in 16 (8\%) cases. All patients had to be readmitted to the acute hospital: 5 of them (2.5\%) with a major bleeding event, $12(75 \%)$ were in a declined systemic condition or had specific reasons (e.g. repeat stroke or epileptic seizure).

\begin{tabular}{|c|c|}
\hline Comorbidities & Number of patients \\
\hline Cardiovascular history & $88(44 \%)$ \\
\hline - vascular disease & 19 \\
\hline - myocardial infarction & 27 \\
\hline - coronary artery disease & 23 \\
\hline - atheroscleosis & 5 \\
\hline Stroke, TIA & $58(29 \%)$ \\
\hline Thromboembolic events & $74(37 \%)$ \\
\hline - Atrial fibrillation & 44 \\
\hline - venous thrombosis & 20 \\
\hline - mechanical valve implantation & 10 \\
\hline General Risk factors for bleeding & $110(55 \%)$ \\
\hline Hypertension & $107(54 \%)$ \\
\hline Gastric ulcer & $3(1.5 \%)$ \\
\hline
\end{tabular}

Table 3: Comorbidities of all 200 patients in the study.

\section{Discussion}

In this study, we analyzed the prescription patterns of APA and OAC including the new substances, in a sample of 200 patients who were consecutively admitted to inpatient rehabilitation after stroke or knee/hip replacement surgery. The average age of our patients was 81.5 years. More than $40 \%(87 / 200)$ of all patients were taking at least 8 different drugs at a time, and $20 \%$ had more than two comorbidities.

Prophylactic NOAC was expectedly prescribed in the arthroplasty sample only, but not for the stroke patients, where the use of VKA was more frequent $(18 / 49=37 \%)$. Dual therapy was less frequently observed in stroke compared to the arthroplasty cases. The most frequent combination was prophylactic NOAC with APA, which means that the higher use of NOAC increases the frequency of dual therapy. We could see that patients with NOAC have more dual therapy. In $5 \%$ of the cases, we found no clear indication for dual anticoagulation with new oral anticoagulation and antiplatelet therapy. One reason may be that neither guidelines nor evidence for double or triple anticoagulation with the new agents exist to date and must be selected individually [21].

In our setting, only $65 \%$ of the patients treated with dual therapy received a proton pump inhibitor, implying the high bleeding risk was not appreciated in every third patient. Yet, two of three patients with a high bleeding risk (HAS-BLED score of $\geq 3$ ) did not receive a PPI. Sixteen patients had to be readmitted to the acute hospital, of whom five patients had a bleeding event (cerebral, gastric or surgical wound). Renal function plays a pivotal role in the prescription of NOAC. Interestingly, renal function was considered in most cases, but the consequent steps of dose adaptation or change of the OAC therapy were not taken. Two patients had a clearance of less than $30 \mathrm{ml} / \mathrm{min}$, but no patient below $15 \mathrm{ml} / \mathrm{min}$ received rivaroxaban, as recommended by the instructions for the use of this drug. Bacchus and Schulmann recommend an annual screening of the creatinine clearance in a long-term use of new oral anticoagulation [22].

It is not only poly-medication that may be a problem for the elderly population, but also under-treatment [23]. In our study, 10 patients (5\%) with documented atrial fibrillation, attaining an average CHA2DS2-VASc score of 3.7 ( $\geq 1$ qualifies for OAC) did not receive anticoagulation therapy. These patients had no absolute contraindications (average HAS-BLED score of $1.7 \pm 0.64$ ).

Our study has several limitations: The total number of 200 patients raises limitations because some combinations were observed in small numbers $<10$. Furthermore, results of the rehabilitation setting cannot be transferred to those patients found in acute hospitals and in outpatient centers. Assessing only the records, but not the patients themselves, may give rise to biases, although one would expect an even higher number of co-diagnoses and co-medications. The study observation time was short and further complications after the rehabilitation period were not assessed.

It is rather obvious that 'real-life' patients are more heterogeneous and polymorbid, and therefore not comparable with a study population. The information from our sample-serving to some point as a quality control-may add some daily clinical experience to the management of anticoagulation therapy, which may become even more complex with further NOACs to come.

Frequent use of NOAC has been observed in the orthopedic subsample. Dual therapy was present in a high proportion of the settings and associated with the use of NOAC. Such a prescription pattern, in combination with frequent comorbidities, elevates the risk of bleeding in this vulnerable elderly population. While the upper gastro-intestinal tract may be protected by the addition of PPI, a significant proportion of the study population cases did not receive any.

\section{Conclusion}

As our study shows, the continuous education of health care providers according to national and international guidelines $[21,24]$ is important to minimize the risk of complications due to anticoagulant treatment in the elderly population. Especially in the setting of new upcoming agents, physicians have to keep these guidelines in mind. Our real-life study in elderly, polymorbid patients with polymedication shows the importance of a critical and regular assessment of indications, interactions, and risks before an anticoagulation therapy is changed or newly combined.

\section{Conflicts of Interests}

All authors report no conflict of interest with the above work.

\section{Acknowledgement}

HS participated in the data collection, performed the statistical analysis and drafted the manuscript. FA participated in the design and coordination of the study, performed the statistical analysis and worked on the manuscript. SL participated in the data collection. AG, JB and AA designed the study, participated in its coordination, and worked on the manuscript. All authors read and approved the final version of the manuscript. 
Citation: Schmidt H, Angst F, Gantenbein AR, Lehmann S, Aeschlimann A, et al. (2016) Practical Experience with Anticoagulation Therapy in an Elderly Polymorbid Rehabilitation Population: What did we Learn?. J Gerontol Geriatr Res 5: 301. doi:10.4172/2167-7182.1000301

Page 5 of 5

\section{References}

1. Pruthi RK (2013) Review of the American College of Chest Physicians 2012 Guidelines for Anticoagulation Therapy and Prevention of Thrombosis. Seminars in hematology 50: 251-258.

2. Brieger D (2014) Anticoagulation: a GP primer on the new oral anticoagulants. Australian family physician 43: 254-259.

3. Russell RD, Hotchkiss WR, Knight JR, Huo MH (2013) The efficacy and safety of rivaroxaban for venous thromboembolism prophylaxis after total hip and total knee arthroplasty. Thrombosis 2013: 762310.

4. Beyer-Westendorf J, Lutzner J, Donath L, Tittl L, Knoth H, et al. (2013) Efficacy and safety of thromboprophylaxis with low-molecular-weight heparin or rivaroxaban in hip and knee replacement surgery: findings from the ORTHO-TEP registry. Thrombosis and haemostasis 109: 154-163.

5. Amin AP, Bachuwar A, Reid KJ, Chhatriwalla AK, Salisbury AC, et al. (2013) Nuisance bleeding with prolonged dual antiplatelet therapy after acute myocardial infarction and its impact on health status. J Am Coll Cardiol 61: 2130-2138.

6. Oldgren J, Wallentin L, Alexander JH, James S, Jönelid B, et al. (2013) New oral anticoagulants in addition to single or dual antiplatelet therapy after an acute coronary syndrome: a systematic review and meta-analysis. Eur Heart J 34: 1670-1680.

7. Huber K, Bates ER, Valgimigli M, Wallentin L, Kristensen SD, et al. (2014) Antiplatelet and anticoagulation agents in acute coronary syndromes: What is the current status and what does the future hold? Am Heart J 168: 611-621.

8. Warle-Van Herwaarden MF, Roukens M, Pop GA, Lamfers EJ, De Smet PA, et al. (2014) Adherence to guidelines for the prescribing of double and triple combinations of antithrombotic agents. Eur J Prev Cardioly 21: 231-243.

9. Hicks KA, Stockbridge NL, Targum SL, Temple RJ (2011) Bleeding Academic Research Consortium consensus report: the Food and Drug Administration perspective. Circulation 123: 2664-2665.

10. Charlot M, Grove EL, Hansen PR, olesen JB, Ahlehoff, et al. (2011) Proton pump inhibitor use and risk of adverse cardiovascular events in aspirin treated patients with first time myocardial infarction: nationwide propensity score matched study. BMJ 342: 2690.

11. Holster IL, Valkhoff VE, Kuipers EJ, Tjwa ET (2013) New oral anticoagulants increase risk for gastrointestinal bleeding: a systematic review and meta-analysis. Gastroenterology 145: 105-112.

12. Beck S, Geser C, Grob D (2012) Multidimensionales geriatrisches Assessment als klinischer Zugang zum multimorbiden Patienten im Spital. PRAXIS 101: 1627-1632.
13. Kongkaew C, Hann M, Mandal J, Williams SD, Metcalfe D, et al. (2013) Risk factors for hospital admissions associated with adverse drug events. Pharmacotherapy 33: 827-837.

14. Howard RL, Avery AJ, Slavenburg S, Royal S, Pipe G, et al. (2007) Which drugs cause preventable admissions to hospital? A systematic review. British journal of clinical pharmacology 63: 136-147.

15. Nickel CH, Ruedinger JM, Messmer AS, Maile S, Peng A, et al. (2013) Drug-related emergency department visits by elderly patients presenting with non-specific complaints. Scand J Trauma Resusc Emerg Med 21:15.

16. Vonbach P, Dubied A, Krahenbuhl S, Beer JH (2008) Prevalence of drugdrug interactions at hospital entry and during hospital stay of patients in internal medicine. Eur J Intern Med 19: 413-420.

17. Graf L, Tsakiris DA (2012) Anticoagulant treatment: the end of the old agents? Swiss medical weekly 142: w13684.

18. Holy EW, Beer JH (2014) Direct oral anticoagulants in the management of venous thromboembolism--evidence from major clinical trials. Seminars in hematology 51: 131-138.

19. Lip GY, Frison L, Halperin JL, Lane DA (2011) Comparative validation of a novel risk score for predicting bleeding risk in anticoagulated patients with atrial fibrillation: the HAS-BLED (Hypertension, Abnormal Renal/ Liver Function, Stroke, Bleeding History or Predisposition, Labile INR, Elderly, Drugs/Alcohol Concomitantly) score. J Am Coll Cardiol 57: 173-180.

20. Naccarelli GV, Panaccio MP, Cummins G, Tu N (2012) CHADS2 and CHA2DS2-VASc risk factors to predict first cardiovascular hospitalization among atrial fibrillation/atrial flutter patients. Am J Cardiol 109: 1526-1533.

21. De Caterina R, Husted S, Wallentin L, Andreotti F, Arnesen H, et al. (2012) New Oral Anticoagulants in Atrial Fibrillation and Acute Coronary Syndromes: ESC Working Group on Thrombosis-Task Force on Anticoagulants in Heart Disease Position Paper. J Am Coll Cardiol 59: 1413-1425.

22. Bacchus F, Schulman S (2015) Clinical experience with the new oral anticoagulants for treatment of venous thromboembolism. Arteriosclerosis, thrombosis, and vascular biology 35: 513-519.

23. Tulner LR, Van Campen JP, Kuper IM, Gijsen GJ, Koks CH, et al. (2010) Reasons for undertreatment with oral anticoagulants in frail geriatric outpatients with atrial fibrillation: a prospective, descriptive study. Drugs \& aging 27: 39-50.

24. Camm AJ, Lip GY, De Caterina R, Savelieva I, Atar D, et al. (2012) 2012 focused update of the ESC Guidelines for the management of atrial fibrillation: an update of the 2010 ESC Guidelines for the management of atrial fibrillation--developed with the special contribution of the European Heart Rhythm Association. Europace 14: 1385-1413. 